\title{
Coronary Artery Disease Risk Assessment in Sedentary and Active Patients: Medical Risk, Behavioral Psychology, and the Standard of Care
}

\author{
Vinay Mehta, $M D^{1}$ and Timothy E Paterick, MD, JD ${ }^{2^{*}}$ \\ ${ }^{1}$ Western Connecticut Health Network, Danbury, CT, USA \\ ${ }^{2}$ Aurora BayCare Medical Center, Green Bay, WI, USA
}

*Corresponding author: Timothy E Paterick, MD, JD, Aurora BayCare Medical Center, Green Bay, WI, USA

\begin{abstract}
Objective: A practical approach to determining risk in sedentary and active patients for coronary artery disease. Identify how personal/family history, atherogenic risk factors, and coronary calcium are essential to determining and predicting risk potential.

Materials/methods: Review of the medical, behavioral psychology, and standard of care literature to identify how human psychology, statistical risk of coronary disease, and coronary calcium shape risk prediction.

Results: A comprehensive personal/family history, risk factor assessment and comprehensive physical examination are the foundation of risk assessment. Understanding the cognitive process of risk potential is critical to management strategies. Stress testing and coronary calcium scoring are useful adjuncts when initial screening is suggestive of intermediate atherogenic risk.

Conclusion: Comprehensive personal/family history, risk factor assessment, comprehensive physical examination, cognitive processing of risk potential, stress testing and calcium scoring all have a role in risk assessment of sedentary and active patients.
\end{abstract}

\section{Keywords}

Coronary artery calcium, Risks factors, Behavioral psychology, Robust exercise, Sedentary, counselling, Standard of care

\section{Introduction}

Today, in the United States, there is a strong public policy to "nudge" patients across all health care disciplines, to an active lifestyle to improve overall health and well-being. In the last several years we have seen an outpouring of work on behavioral economics, behaviorally informed policies, and "nudges," understood as interventions that encourage people to assume behaviors that presumably improve short and long-term health. This "nudging" includes the disclosure of important information about the risks of smoking, atherogenic diet, excess alcohol, and physical inactivity from the best evidence literature [1]. The rationale for the "nudges" is that robust physical activity and habitual exercise training are associated with a reduction in cardiovascular events [1-3] and increased longevity [4]. The reason for the mounting interest in "nudges" should not be obscure; nations seek to address mounting social problems such as tobacco abuse, excess alcohol use, and obesity with tools that work and do not cost a great deal $[5,6]$.

The promotional, health "nudges" are supported by the fact that low level physical activity (sedentary patients) is responsible for $12 \%$ of cardiovascular mortality [7]. Inferentially, exercise is one of the principle modality to reduce the risk of coronary heart disease and potentially cardiovascular premature death.

Succinctly stated, the credo and teachings of the health care community have been that exercise results in health benefits, but recent medical investigations have created pause for physicians, causing them to reconsider the exercise dogma. The pause occurred because recent studies suggest long-term and high intensity exercise may actually increase the prevalence and severity of atherosclerotic heart disease among mid-

Citation: Mehta V, Paterick TE (2020) Coronary Artery Disease Risk Assessment in Sedentary and Active Patients: Medical Risk, Behavioral Psychology, and the Standard of Care. Int Arch Cardiovasc Dis 4:032. doi.org/10.23937/2643-3966/1710032

Accepted: October 10, 2020: Published: October 12, 2020

Copyright: (C) 2020 Mehta V, et al. This is an open-access article distributed under the terms of the Creative Commons Attribution License, which permits unrestricted use, distribution, and reproduction in any medium, provided the original author and source are credited. 
dle-aged patients $[8,9]$. The unknown is whether this finding of increased coronary atherosclerosis predicts adverse cardiovascular outcomes.

These investigations unexpectedly revealed that there was a high prevalence of coronary artery calcifications and plaque in active patients. If we assume coronary calcification and plaque burden increase the risk for adverse cardiac events, these findings conflict with the notion that robust physical activity and habitual exercise training is associated with a reduction in cardiovascular events.

The paradigm predicament described above demands an inquiry into the following questions:

- What does coronary artery calcification and plaque architecture mean for patients and does it have the potential for adverse outcomes?

- Are there gender related differences in the meaning of coronary artery calcifications and risk for adverse cardiovascular events?

- How do physicians understand and counsel patient intuitions, fears and stressors related to adverse cardiovascular events?

- How do physicians evaluate the safety of patients who engage in robust physical activity and who have been identified to have a burden of coronary artery calcification?

- How do physicians evaluate the safety of patients with significant comorbidities, coronary artery calcification, but a lifestyle lacking in physical activity/exercise?

This manuscript will address the patient and physician concerns when evaluating the risks of actively exercising and sedentary behavior in patients with coro- nary artery calcification, the cognitive risk assessment applied by patients and physicians when addressing the risk of exercise and inactivity, whether the level of exercise plays a role in developing coronary artery calcifications, whether there are gender differences that play a role in the development of coronary calcifications, how physicians should medically evaluate and counsel both active and inactive patients, and whether the law impacts physician decision-making.

\section{Vigorous Exercise - Intuitions and Probability:} Lessons from Behavioral Psychology and Economics

It is understandable that patients often lack, and struggle to understand, risk related information. They typically do not think about the nature and magnitude of risk at issue, and they may know little about the various consequences of risk reduction. Often assessment of risk, especially when the risk includes death, is handled with emotions and intuitive thinking. Most exercise facilities typically have the visually salient nudge: "Ask Your Doctor before Starting Any Exercise Program" (Figure 1).

The "availability heuristic" often plays a role in patient assessment of risk. Patients' judgments about probability are often affected by whether a recent negative event is cognitively "available", prompting an individual to overestimate the risk [10-12]. If a risk is not cognitively available, they may well underestimate the risk (probability neglect). With respect to risks (smoking, food gorging, sedentary lifestyle), judgments are typically affected by the "availability heuristic". An obese relative who smokes and dies, suddenly impacts how patients think about obesity and smoking. In the aftermath of an earthquake, the purchase of earthquake in-

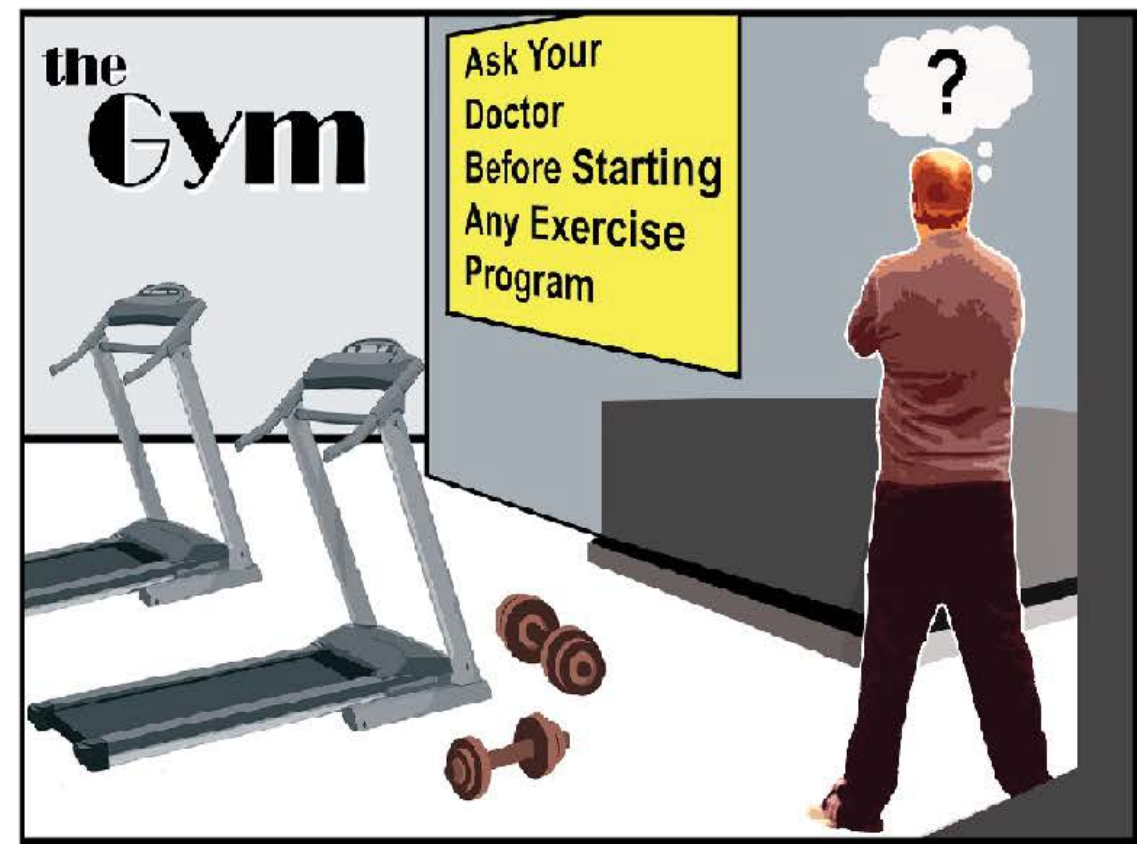

Figure 1: Vigorous exercise. 


\section{System I Thinking}

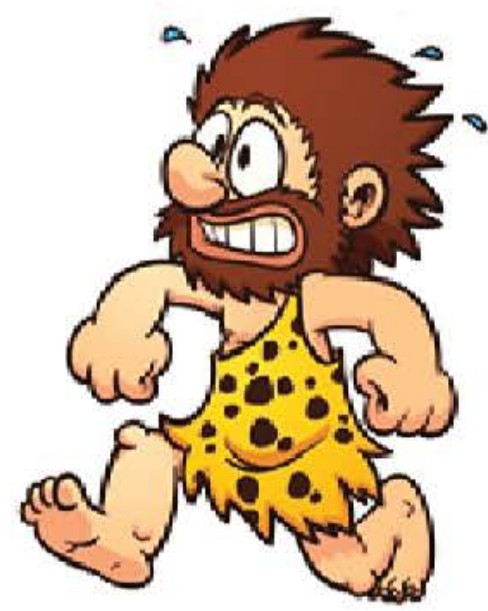

Fight or Flight Response: Anxietyifear

\section{System II Thinking}

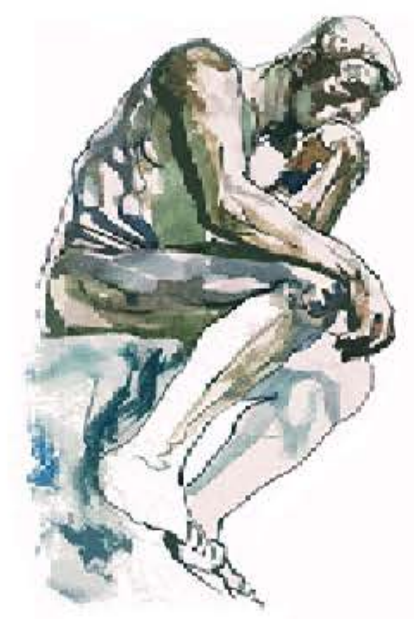

ThoughtfuliDeliberate

Figure 2: Human mind has two cognitive systems, System I and System II.

surance skyrockets due to the salience of earthquakes, but it declines steadily as vivid memories recede. This same "availability heuristic" applies when a high-profile athlete dies on the athletic field, or when a relative or friend experiences a myocardial infarction, coronary artery bypass grafting, or sudden cardiac death. These points suggest that personal or highly publicized events are likely to lead to people being exceedingly fearful of statistically small risks; example: 9/11 and terrorism. Emotions play a large role; if people can visualize the "worst case scenario," assessment of true probabilities will be crowded out by fear. There is a lesson here about how to attract public attention to a risk: Make a vivid example of its occurrence highly salient to the community.

\section{Understanding Risk Appraisal by the Commu- nity - Two Systems in the Mind}

How do people respond to potential health risk? This is an important question that requires an in-depth exploration to help physicians develop strategies for counseling patients when assessing risk of adverse cardiovascular events. Social science describes the human mind as having two cognitive systems, System I and System II. System I is the automatic system, while System II is more deliberative and reflective. System I response occur fast. It is emotional and intuitive, responding on automatic pilot. It is driven by habits and is often referred to as "amygdala hijacking". It has a lot of trouble with complexity. System II is deliberative and calculating. It thinks and considers probability calculations carefully and slowly-reflecting due diligence. It is a planner rather than a doer and can handle complexity. System II recognizes we need to make trade-offs, and that we will, inevitably, be making decisions about the extent to employ various resources to understand and hopefully reduce risks, especially mortality risks (Figure 2).
A great deal of work suggests that people evaluate risks and benefits through the use of the "availability heuristic" and through the "affect heuristic". When the "affect heuristic" is at work, people assess benefits, costs, and probabilities not by running the numerical statistics, but by consulting their feelings (gut check). System I is doing all the work here.

What actually are these two Systems? In the case of cognitive conflict, which adjudicates? The best answer is that the idea of two systems is a simplification that is designed to distinguish automatic, effortless cognitive processing and more complex, effortful cognitive processing. System II must be executed when we try to assess whether the long-term benefits of an action (diagnostic testing, restricted/unrestricted activity) outweigh the long-term physical and financial costs. Type II thinking is critical when assessing the pre-test and post-test probabilities of an outcome and the benefits and risks of promoting or restricting activity, in physically active and sedentary patients.

\section{Does Physical Activity Make a Patient Prone or Protect against the Development of Coronary Atherosclerosis?}

There are two related questions to address: Does robust exercise promote or reduce coronary artery calcification? The follow up question is even more important, if it promotes calcification does that calcification result in adverse cardiac events?

The development and progression of coronary artery disease is dependent upon many variables including phenotype to genotype expression, diet/nutrition and physical activity [7]. Atherosclerosis is typically considered a disease of modern society that is composed of atherogenic diets and physical inactivity. A post-mor- 
tem pathology study evaluated coronary atherosclerosis in men aged 30-60 years who died suddenly from accidents, homicide and suicide [13]. This autopsy study designated the deceased patients as sedentary or physically active, based upon their occupations. The surprising and unexpected finding was that there was a similar degree of coronary atherosclerosis in the two groups. The findings were in contradistinction to the widespread convictions that exercise is associated with less coronary artery disease and longevity. The inference from this study was coronary atherosclerosis was independent of the level of physical activity [13]. To add fuel to the fire of this controversy, a case study of competitive, elite marathon runners revealed none of the runners had atherosclerosis, raising a conjecture that marathon runners may be immune to coronary atherosclerosis as they avoided tobacco, ate healthy diets, and habitually performed high levels of physical activity [14]. A contradictory small study series of 4 marathon runners who had died disclosed that all had coronary atherosclerosis, raising doubt to the hypothesis that running resulted in immunity to the development of coronary atherosclerosis [15].

These incongruous findings suggested that high levels of physical activity did not create immunity to coronary atherosclerosis. This dichotomy raised a concern as to how physicians should counsel patients engaged in vigorous physical activity and whether the medical community should even "nudge" sedentary patients to exercise.

\section{How Do We Non-Invasively Assess Coronary Atherosclerosis in Patients?}

We have new tools that do not require an autopsy or invasive procedures to assess coronary atherosclerosis in patients. Today coronary atherosclerosis is often measured non-invasively using a CT scan with or without contrast. A non-contrast CT scan allows quantification of coronary artery calcium by calculating a coronary artery calcium score (CAC score) [16].

The Agatston CAC score is calculated by multiplying the area of calcification by 1 through 4 depending on the density of the area, and summing the scores of all slices. The density score is based upon the highest Hounsfield unit (HU) of the area with a density of score of 1 for $\mathrm{HU}$ 130-199, 2 for HU 200-299, 3 for HU 300-399, and 4 for HU 400 [17] (Table 1). CAC scoring only includes areas with a density $>130 \mathrm{HU}$ and $>1 \mathrm{~mm}^{2}$. The CAC scoring is related to the calcium burden - a predictor for cardiovascular disease (Table 2).

This model attempts to use CAD scoring as a surrogate for autopsy data to assess atherosclerotic burden and the potential risk for cardiovascular morbidity and mortality. The CAC score is the product of CAC area and density. Agatson score is most commonly used to risk stratify patients, but there are some studies which have shown that higher CAC volume is associated with higher cardiovascular disease risk, whereas; higher CAC density is associated with lower risk, [18] thus giving us pause that a higher calcium score may not necessarily have a linear adverse correlation.

Contrast enhanced coronary CT scanning (CTTA) allows the lumen of the coronary arteries to be imaged with precise assessment of plaque characteristics. This allows the separation of plaques into categories:

- Calcified plaque

- Non-calcified plaque

- Mixed calcified and non-calcified plaques

This classification allows potential prediction of cardiovascular risk $[18,19]$. CCTA assesses the degree of risk based upon the morphological features of the plaque with higher risk features being: 1) The plaque demonstrating the "napkin ring sign", 2) Evidence of positive remodeling of the plaque, 3) Evidence of low attenuation plaque, and 4) Spotting calcification of the plaque. These morphological features are all associated with adverse prognosis [19].

Despite the potential benefit of CAC scoring and CCTA, it must not be used indiscrimately. Because the use of CCTA uses radiation we must be certain that implementation requires careful assessment of the value of CCTA to the individual patient [20].

Is There a Defining Distinction between Atherosclerotic Plaque Characteristics in Sedentary and Active Patients?

As discussed above, there are studies that support the idea that active patients are not immune to coronary atherosclerosis $[8,21,22]$, in contradistinction to the generally accepted assumption that coronary atherosclerosis would be less prevalent in physically active patients compared to a sedentary population. Collectively these studies suggest that calcium scores in robustly exercising patients may have comparable calcium

Table 1: Agatston CAC score.

\begin{tabular}{|l|l|}
\hline Hounsfield Unit of area & Density score \\
\hline $130-199$ & 1 \\
\hline $200-299$ & 2 \\
\hline $300-399$ & 3 \\
\hline$>400$ & 4 \\
\hline
\end{tabular}

Table 2: Calculating the risk of cardiovascular by comparing CAC score.

\begin{tabular}{|l|l|}
\hline CAC score & Rate of cardiovascular Events \\
\hline 0 & $2.1 \%$ \\
\hline $1-100$ & $12.9 \%$ \\
\hline$>100-400$ & $16.3 \%$ \\
\hline$>400$ & $33.8 \%$ \\
\hline
\end{tabular}


scores to sedentary controls. This is perplexing given the fact that physical exercise and habitual exercise training are associated with reduced cardiovascular events and increased longevity [1-4]. These findings surface cognitive conflict regarding the role of exercise and risks associated with CAC scoring.

Examining the variation in plaque morphology identified in these two groups is the best way to rectify this discrepancy. Investigators identified a distinct difference in plaque morphology between the active and sedentary groups. Physically active patients had calcified plaque while the sedentary group had mostly mixed (calcified and non-calcified) plaques [23]. This raises the possibility that plaque morphology may be a discriminator between active patients and sedentary controls and may have implications for risk prediction [8]. The distinction between calcified plaque and mixed plaque appears to be critical to understanding how there can be an elevated CAC score in both physically active patients and sedentary patients, yet a difference in risk for adverse cardiac events.

This distinction is critical in our attempt to manage risk in active and sedentary patients of both genders. The gender difference is enigmatic and needs further investigation. The majority of patients in current studies have been males. This may be related to the fact that males have a higher prevalence of coronary atherosclerosis and a higher risk of sudden death during exercise [24]. Limited evidence is available, but current data suggest that the association between exercise and coronary atherosclerosis is weaker in active females compared to male athletes [25]. This needs further investigation.

\section{Does Determination of Coronary Artery Calci-} fication Influence Medical Counseling?

A typical medical assessment includes a detailed personal and family history and a comprehensive physical examination. Based upon the history and physical examination, the physician estimates a pre-test probability of coronary artery disease (Bayesian analysis), determining if an ECG and a stress test are indicated. This approach is compatible with the accepted national standard of care.

In a 2016 study, 318 middle-aged male, amateur athletes underwent a sports medical evaluation without any abnormalities identified in the history and physical examination. CT scanning and CCTA testing revealed $19 \%$ had a calcium score $>100$ and/or $>50 \%$ stenosis of a coronary artery [26]. This raises the query whether asymptomatic active patients may have subclinical coronary atherosclerosis that is not detected during routine sports medical evaluation? If so, are they at increased risk of exercise-induced cardiac events?

Currently, CAC scoring among physically active patients is not recommended as a screening tool. The calculated number needed to screen with CAC scoring plus
CCTA to prevent one cardiovascular event, defined as angina, myocardial infarction, coronary revascularization, resuscitated cardiac arrest, stroke, or cardiovascular death, within 5 years has been studied [26]. The estimated number needed to screen using only CAC scoring was $n=183$ and $n=159$ for CAC scoring plus CCTA. Thus screening with CAC scoring and CCTA lacks feasibility when applying cost benefit analysis. Indiscriminate screening has the potential for unnecessary collateral testing. These indeterminate results put patients in risk purgatory with many unintended physical and psychological effects.

There are remaining unanswered questions regarding the relationship between exercise and coronary atherosclerosis: What is responsible for the development of coronary calcification in physically active patients? Does a similar Agatson score predict differential prognosis for physically active versus inactive individuals? Is there a dose related relationship between exercise and coronary calcification and what does that relationship look like? What role do other comorbidities, family history or ethnicity play in evaluating the coronary artery calcium score for clinical decision making? Further research will be needed to identify the mechanism for the development of coronary calcification and variable plaque morphology to more accurately risk stratify these different groups.

\section{Patients' Fears and Emotional Responses}

Patients frequently bring a mindset of worst-case scenario thinking to the office visit. These patients can be characterized as "worst-case scenario entrepreneurs". Their emotional concerns present to the physician with a zeal and passion that can be hard to curtail. Recent events in their personal lives have surfaced trepidation similar to the aftermath of the attacks on 9/11. For especially horrific outcomes, it is tempting for patients to think that a one percent chance should be treated as a certainty.

Recall that judgment about risks comes through two different pathways [27]. Type I, automatic and Type II, deliberative. Often individual judgment is rooted in individual experience, such as the death of a sibling or a close relative (genetic underpinnings). Additionally, with social media someone else's tragic outcome may have great salience due to high visibility. These are examples of Type I responses. Alternatively, judgments might emerge from Type II processing of statistical accounts of the role of cholesterol levels, exercise regiments, and testing modalities. While patient judgment is affected by both of these cognitive pathways, personal experience is typically far more effective in motivating behavior.

Current evidence suggests that the amygdala - located deep within the temporal lobes of the brain - makes people alert to health danger before the prefrontal cor- 
tex gets involved [28]. The important point is that patients have immediate and often-visceral reactions to presumed, life threatening health related situations, and the immediate reaction operates as a mental short cut before a more deliberative or analytic assessment of the underlying health issues.

A worst-case scenario that triggers System I is particularly likely to influence the conduct of patients. It might paralyze patients into a state of fear of dying. Once System II becomes involved through physician counseling, it hopefully will create a deliberative check, ensuring an eventual conclusion that presumed risks are insignificant. Physicians must be cognizant of the System I and System II thinking understanding patient responses to worst - case scenarios, and in particular their susceptibility to excessive overreaction. Physicians need to help patients think more rationally about low-probability risks of sudden death, cancer, and dementia in this world of social media igniting public salience to these health-related issues. Ironically, extreme emotional responses and intense stresses may be more dangerous that the low risk potential of a worst-case scenario (Figure 2).

Catastrophic Harm (Cardiac Morbidity/Mortality) Precautionary Principle

Physicians must develop strategies to advise both patients with a mindset of worst-case scenario and patients with 'probability neglect', where patient have high risk features for cardiovascular disease (genetic predisposition, hypertension, hyperlipidemia, diabetes, smoking, and obesity) and a high probability for coronary artery disease, but ignore their statistical risk for life threatening events.

We have discussed the asymptomatic, actively exercising patient worst-case scenario thought process in detail. They must be shifted from System I to System II and educated that their level of risk is extremely small and taught that maintaining their present low risk requires adherence to strict risk factor management, heart healthy eating, and routine aerobic exercise.

The 'probability neglect' subset of patients has a high-risk profile through genetics, risks factors, and life style decisions. When risks have catastrophic, worstcase scenarios, it makes sense to take special measures to eliminate those risks. Most worst-case scenarios have an element of irreversibility (death). Special precautions should be taken to avoid irreversible harms, (lifestyle modifications and medications) that go well beyond those that would be taken if irreversibility were not a problem.

Often patients see future medical procedures as "futures options", albeit as their health "insurance". They believe they do not need to alter their current activities because they will have their "futures options" of coronary stenting, coronary bypass surgery, automatic im- plantable defibrillators, and cardiac transplantation. It is imperative that physicians educate patients about the limitations of invoking their "futures options", which have associated risk and limited long-term benefits. Choosing the route of "future options" may adversely affect the patient and the patient's family because of the risks of the procedures and the often limited extent of benefit. These procedures should be limited to a last ditch approach when all attempts at prevention fail.

\section{Does the Law Impact Medical Risk Assessment for Active and Sedentary Patients?}

As in all patients, the law does have a role in management of these patients. The law is a physician's ally when it comes to managing these patients. It demands that physicians meet the standard of care through an adherence to the standards of medical informed consent. Medical informed consent is ethically, morally, and legally mandated by the fiduciary responsibilities flowing from the patient-physician relationship [29].

Physicians have an ethical responsibility to identify the best treatments for each patient on the basis of available medical evidence, and to discuss with patients the potential benefits and risks. They must allow for patients' questions about the proposed treatments, benefits, and risks and must answer those questions from the available medical literature and their professional experience. This exchange of information and ideas is the foundation of the patient-physician partnership and promotes informed decision making in the most complex medical situations. This exchange requires an understanding of System II deliberation to counterbalance System I reactive tendencies when making healthcare related decisions.

Based Upon Medical Probabilities, Behavioral Psychology, and Legal Tenets How Should Physicians Manage Active and Sedentary Patients?

The physician assessment should take into consideration the patient's concerns and medical risks for cardiovascular disease. Even if the patient presents with System I thinking, the physician must evaluate the potential fear, anxiety or misinformation that is leading to the conclusions that the patient has drawn. With empathy, objectivity and in-depth discussion the conversation can be directed to incorporate the objectivity and thoughtfulness of System II thinking (Figure 3).

The evaluation and any proposed treatment must be based on the patient's risk for cardiovascular disease, based upon a comprehensive, personal and family history, and comprehensive physical examination. Through education and choice architecture patients should be counseled and "nudged" to manage risk factors: Including blood pressure, lipid status, blood sugar levels, weight control, and smoking cessation. 


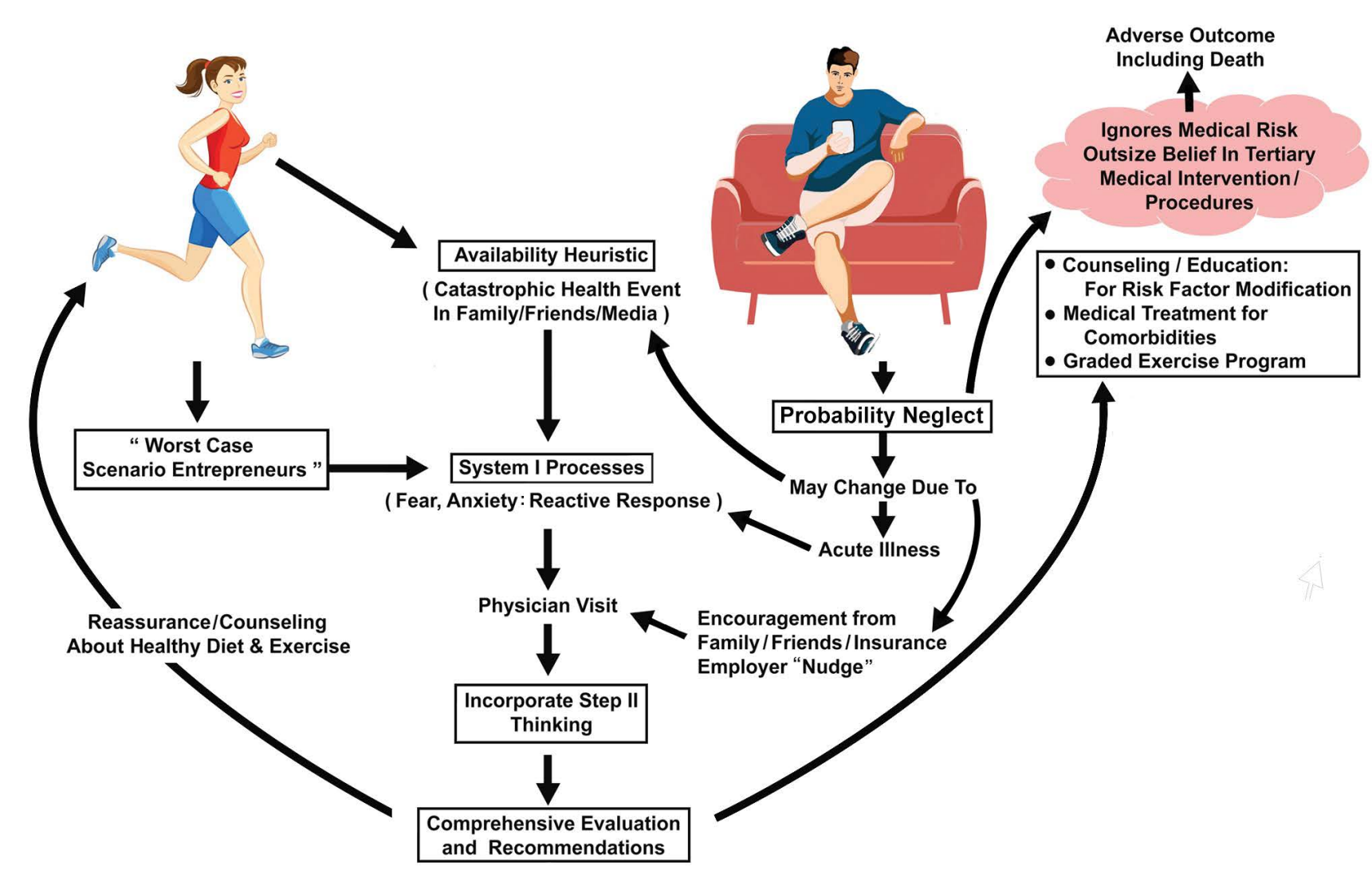

Figure 3: Comprehensive evaluation and proposed treatment.

With either classical symptoms of angina pectoris or atypical symptoms, such as reduced exercise tolerance, shortness of breath, or fatigue, additional testing should be considered. The decision to test depends upon a synthesis of symptoms, risk factors, and physical examination findings used to determine the Bayesian pre-test probability for coronary artery disease. If that Bayesian assessment suggests moderate probability for coronary artery disease, initial testing may include ECG and exercise stress testing similar to the general population.

Although there are no specific guidelines when to pursue CAC scoring or CCTA, these testing modalities should not be used in asymptomatic patients without a high-risk cardiovascular profile. CAC scoring and CCTA use should be carefully considered for high-risk asymptomatic cardiac patients and symptomatic patients. Widespread use of CAC scoring and CCTA has the potential for many unintended physical and psychological consequences.

What about the patient that presents with CAC scoring and CCTA data from the outside? How do physicians proceed? In the setting of asymptomatic patients that are exercising at a robust level, this outside data can be difficult to cognitively process for patients. This makes counseling more difficult as physicians must take the time to educate the patient about the best available medical evidence in relationship to the CAC scoring and CCTA findings. So, how do you treat an asymptomatic, active patient who brings from the outside an elevated CAC score and CCTA results showing plaque? If the plaque is calcified a pragmatic approach is to maximize treatment of risk factors, encourage continued exercise, prudent diet, and the reporting of any symptoms [3032].

If the plaque is non-calcified CCTA is used to evaluate anatomy: including coronary stenosis, plaque morphology, and number and location of plaques. In the setting of stenosis $>50 \%$ and mixed morphology plaque we recommend functional testing for ischemia and again treatment of all risk factors. Although CAC progression is associated with coronary and cardiovascular events; it does not add to risk prediction [33]. Coronary angiography is not recommended in the setting of an asymptomatic patient unless the physician deems the burden of ischemia is large. If ischemia occurs at a certain blood pressure and heart rate level, the patient can be counseled to exercise below that threshold, thus, reducing the potential for ischemia. Currently there is no evidence that stenting will increase life expectancy in asymptomatic patients.

The standard of care requires physicians to educate patients regarding the available data on the benefits of exercise for their cardiovascular health and well-being (Table 3). Physicians must allow for patients' questions about the proposed treatments, benefits, and risks and must answer those questions from the available medical literature and their professional experience. This exchange of information and ideas is the foundation of the patient-physician partnership and promotes informed decision making in the most complex medical 
Table 3: Benefits of exercise for cardiovascular health and well-being.

\begin{tabular}{|c|c|c|c|}
\hline & Active Female & Active Male & Sedentary $M / F$ \\
\hline Obesity & Negative & Negative & Positive/Negative \\
\hline Hypertension & Negative & Negative & Positive \\
\hline Diabetes & Negative & Negative & Positive \\
\hline Hyperlipidemia & Positive/Negative & Positive/Negative & Positive \\
\hline CAC & Negative & Positive/Negative & Positive \\
\hline $\begin{array}{l}\text { Abnormal Plaque } \\
\text { morphology }\end{array}$ & Negative & Negative & Positive \\
\hline Poor nutrition & No & No & Yes \\
\hline Type I thinking & No & No & Yes \\
\hline Type II thinking & Yes & Yes & No \\
\hline Adverse cardiac events & No & No & No \\
\hline Longevity & Yes & Yes & No \\
\hline
\end{tabular}

situations. This conversation should hopefully "nudge" patients to improve their lifestyle decisions to increased physical activity. We must equate present health values and future health values. In the financial world we have a deep understanding of present value, interest rate, and future values. That is why we all invest in our retirement fund, and our country developed a safety net called social security. The question we all must confront is do we invest in a health retirement fund? How much are people willing to invest in their health today to develop a safety net that potentially reduces future health risk?

\section{Conclusion}

Comprehensive personal/family history, risk factor assessment, comprehensive physical examination, cognitive processing of risk potential, stress testing and calcium scoring all have a role in risk assessment of sedentary and active patients.

Generally robust physical activity and habitual exercise training are associated with a reduction in cardiovascular events [1-3] and increased longevity [4]. Despite recent evidence suggesting that robust exercise may result in increased coronary atherosclerosis, patients should be "nudged" to continue to exercise, as exercise has been associated with decreased cardiovascular events and increased longevity.

The apparent paradox of coronary atherosclerosis occurring in active patients not experiencing cardiovascular events may be rectified by the fact that those undergoing CAC scoring and CCTA have increased calcium without malignant plaque morphology. If there is identification of abnormal plaque morphology it may be prudent to pursue functional testing. As we progress scientifically it is essential that we further investigate the underlying mechanisms, clinical relevance, and optimal management of coronary atherosclerosis in asymptomatic active and sedentary adults.

Presently, it is critical to apply System II thinking when considering the management of asymptomatic active and sedentary patients. The available data strongly supports physical activity and "nudging" all patients after comprehensive history and physical examination, to engage in risk factor modification and daily physical activity. Doing so, while practicing informed consent, should meet the standard of care and limit any potential for allegations of negligence.

\section{Acknowledgements}

Acknowledgement of Brian Schurrer or support for the art work and illustrations.

\section{References}

1. Lee DC, Pate RR, Lavie CJ, Sui X, Church TS, et al. (2014) Leisure-time running reduces all-cause and cardiovascular mortality risk. J Am Coll Cardiol 64: 472-481.

2. Eijsvogels TM, Thompson PD (2015) Exercise is medicine: At any dose? JAMA 314: 1915-1916.

3. Wen CP, Wai JP, Tsai MK, Yang YC, Cheng TY, et al. (2011) Minimum amount of physical activity for reduced mortality and extended life expectancy: A prospective cohort study. Lancet 378: 1244-1253.

4. Lee DC, Brellenthin AG, Thompson PD, Sui X, Lee IM, et al. (2017) Running as a key lifestyle medicine for longevity. Prog Cardiovasc Dis 60: 45-55.

5. Richard H Thaler, Cass R Sunstein (2008) Nudge: Improving decisions about Health, Wealth and Happiness.

6. David Halpern (2015) Inside the nudge unit: How small changes can make a big difference.

7. Benjamin EJ, Virani SS, Callaway CW, Chamberlain AM, Chang AR, et al. (2018) Heart Disease and Stroke Statistics - 2018 update: A report from the American Heart Association. Circulation 137: e67-e492.

8. Aengevaeren VL, Mosterd A, Braber TL, Prakken NH, Doevendans PA, et al. (2017) Relationship between lifelong exercise volume and coronary atherosclerosis in athletes. Circulation 136: 138-148.

9. Merghani A, Maestrini V, Rosmini S, Cox AT, Dhutia H, et al. (2017) Prevalence of subclinical coronary artery disease in masters endurance athlete with a low atherosclerotic risk profile. Circulation 136: 126-137. 
10. Paul Slovic, Baruch Fischloff, Sarah Lichtenstein (2000) "Cognitive Processes and Social Risk Taking", in the Perception of Risk. London: Earth-scan 32: 37-38.

11. Daniel Kahneman (2011) Thinking Fast and Slow. Farrar, Straus and Giroux, New York.

12. Paul Slovic (2010) The feeling of risk: New perspectives on risk perception. Taylor \& Francis, New York.

13. Spain DM, Bradess VA (1960) Occupational physical activity and the degree of coronary atherosclerosis in "normal" men. A postmortem study. Circulation 22: 239-242.

14. Bassler TJ (1977) Marathon running and immunity to atherosclerosis. Ann NY Acad Sci 301: 579-592.

15. Noakes TD, Opie LH, Rose AG, Kleynhans PH, Shepers NJ, et al. (1979) Dowdeswell R. Autopsy-proven coronary atherosclerosis in marathon runners. NEJM 301: 86-89.

16. Blaha MJ, Mortensen MB, Kianoush S, Tota-Mahara R, Cainzos-Achirica M (2017) Coronary artery calcium scoring: is it time for a change in methodology? JACC Cardiovasc Imaging 10: 923-937.

17. Agatston AS, Janowitz WR, Hildner FJ, Zusmer NR, Viamonte M Jr, et al. (1990) Quantification of coronary artery calcium using ultrafast computed tomography. J Am Coll Cardiol 15: 827-832.

18. Criqui MH, Denenberg JO, Ix JH, McClelland RL, Wassel CL, et al. (2014) Calcium density of coronary artery plaque and risk of incident cardiovascular events. JAMA 311: 271278.

19. Nerlekar N, Ha FJ, Chesire C, Rashid H, Cameron JD, et al. (2018) Computed tomographic coronary angiographyderived plaque characteristics predict major adverse cardiovascular events: A systemic review and meta-analysis. Circ Cardiovasc Imaging 11: e006973.

20. Paterick TE, Jan MF, Paterick ZR, Tajik AJ, Gerber TC (2012) Cardiac imaging modalities with ionizing radiation: The role of informed consent. JACC Cardiovasc Imaging 5: 634-640.

21. Defina LF, Radford NB, Barlow CE, Willis BL, Leonard D, et al. (2019) Association of all-cause mortality and cardiovascular mortality with high levels of physical activity and concurrent coronary artery calcification. JAMA Cardiol 4: 174-181.

22. Aengevaeren VL, Mosterd A, Braber TL, Sharma S, Thompson PD, et al. (2019) Coronary atherosclerosis in athletes: exploring the role of sporting discipline. JACC Cardiovasc Imaging 12: 1587-1589.

23. Hou ZH, Lu B, Gao Y, Jiang SL, Wang Y, et al. (2012) Prognostic value of coronary CT angiography and calcium score for major adverse cardiac events in outpatients. JACC Cardiovasc Imaging 5: 990-999.

24. BerdowskiJ, de Bues MF, Blom M, Bardai $A$, Bots ML, et al. (2013) Exercise-related out-of-hospital cardiac arrest in the general population: Incidence and prognosis. Eur Heart J 34: 3616-3623.

25. Roberts WO, Schwartz RS, Kraus SM, Schwartz JG, Peichel G, et al. (2017) Long-term marathon running is associated with low coronary plaque formation in women. Med Sci Sports Exer 49: 641-645.

26. Barber WO, Moster A, Prakken NH, Rienks R, Nathoe HM, et al. (2016) Occult coronary disease in middle-aged sportsman with a low cardiovascular risk score: The Measuring Athlete's Risk of Cardiovascular Events (MARC) study. Eur Heart J Prev 23: 1677-1684.

27. Elke Weber (2006) Experience-based and description-based perception of long-term risk: Why global warming does not scare us (Yet). Climate Change 103.

28. Joseph E LeDoux (1996) The Emotional Brain. Simon \& Schuster, New York.

29. Timothy J Paterick, Geoff $\vee$ Carson, Marjorie C Allen, Timothy E Paterick (2008) Medical Informed Consent: General Considerations for Physicians. Mayo Clin Proc 83: 313-319.

30. Simon C Mathews, David L Narotsky, David L Bernholt, Matthew Vogt, Yu-Hsiang Hsieh, et al. (2012) Mortality Among Marathon Runners in the United States, 2000-2009. Am J Sports Med 40: 1495-1500.

31. US Public Health Service (2008) Physical Activity Guidelines Advisory Committee report, 2008. US Department of Health and Human Services, Washington (DC).

32. Keith M Diaz, Virginia J Howard, Brent Hutton, Natalie Colabianchi, John E Vena, et al. (2017) Patterns of sedentary behavior and mortality in US middle-aged and older adults: A national cohort study. Ann Intern MED 167: 465-475.

33. Nils Lehmann, Raimund Erbel, Amir A Mahabadi, Michael Rauwolf, Stefan Möhlenkamp, et al. (2018) Value of Progression of Coronary Artery Calcification for Risk Prediction of Coronary and Cardiovascular Events. Circulation 137: 665-679. 